S. Suihkonen, J. Sormunen, V. T. Rangel-Kuoppa, H. Koskenvaara and M. Sopanen, Growth of InN by vertical flow MOVPE, Journal of Crystal Growth 291, 8-11 (2006).

(C) 2006 Elsevier Science

Reprinted with permission from Elsevier. 


\title{
Growth of InN by vertical flow MOVPE
}

\author{
S. Suihkonen*, J. Sormunen, V.T. Rangel-Kuoppa, H. Koskenvaara, M. Sopanen \\ Optoelectronics Laboratory, Helsinki University of Technology, P.O. Box 3500, FIN-02150 HUT, Finland
}

Received 28 February 2005; received in revised form 15 December 2005; accepted 20 February 2006

Communicated by C.R. Abernathy

\begin{abstract}
InN films were grown by metal-organic vapour phase epitaxy (MOVPE). The growth was performed in a MOVPE apparatus with a vertical reactor geometry optimized for the growth of $\mathrm{GaN}$. The reactor geometry is found to cause enhanced $\mathrm{cracking}$ of $\mathrm{NH}_{3}$, and the growth rate is limited by the amount of reactive indium in the temperature range of $550-650{ }^{\circ} \mathrm{C}$. The grown films are characterized comprehensively. Experimental results indicate that the InN films, grown on sapphire substrates, contain metallic indium and the film surface consist of hexagonal islands. Growth temperature has a strong effect on the surface island size, optical quality and electrical properties of the InN layer. The desorption of nitrogen is assumed to cause the formation of metallic indium above $550{ }^{\circ} \mathrm{C}$.
\end{abstract}

(C) 2006 Elsevier B.V. All rights reserved.

PACS: 81.15.Gh; 81.05.Ea

Keywords: A3. Metalorganic chemical vapour deposition; B1. Indium nitride; B2. Semiconducting III-V materials

\section{Introduction}

During the last few years, development in the field of IIInitrides has been fast. Current GaN-based device technologies include light emitting diodes (LEDs), laser diodes (LDs), UV detectors, and ultrahigh power switches. Indium is used in the active InGaN layers of GaN LEDs and LDs to extend the emission of $\mathrm{GaN}$ based materials to longer wavelengths. Due to the difficulties in growth, the indium content in InGaN alloys is low. Pure indium nitride is the least known of the III-N materials. Until recently, the commonly quoted value for the optical band gap energy of InN was $1.89 \mathrm{eV}$, but new measurements of high quality InN films have shown evidence of a much smaller band gap between 0.65 and $0.90 \mathrm{eV}$ [1]. This smaller band gap is compatible with the main wavelength range of optical communication. Additionally, InN has excellent electron transport properties as predicted theoretically [2]. Combining high quality InN films with existing $\mathrm{GaN}$ technology would open new possibilities in the field of optical telecommunication and electronics.

\footnotetext{
${ }^{*}$ Corresponding author. Tel.: +35894512325 ; fax: +35894513128 .

E-mail address: sami.suihkonen@hut.fi (S. Suihkonen).
}

The growth of high quality InN films has proven to be difficult. This is mainly because of the small growth temperature window. The growth temperature is limited from above by the desorption of nitrogen and thermal decomposition of the films [3]. From below the growth temperature is limited by low thermal decomposition rate of ammonia $\left(\mathrm{NH}_{3}\right)$ [4], so a high $\mathrm{V} / \mathrm{III}$ ratio is needed. The formation of metallic indium is a well-known problem caused by either the lack of reactive nitrogen or the desorption of nitrogen [5].

Reactor geometry and design have been found to be critical factors in metal-organic vapour phase epitaxy (MOVPE) growth of $\mathrm{GaN}$ and $\mathrm{InN}[6,7]$. The influence of reactor geometry on GaN growth has been systematically studied and MOVPE reactors optimized for growth of $\mathrm{GaN}$ are commercially available. Compared to $\mathrm{GaN}$, little information on the effect of reactor geometry on the growth of $\mathrm{InN}$ is available. Separate reactant gas delivery of group III and V precursors close to the substrate has been found to prevent harmful pre-reactions in the growth of all III-N materials. High gas velocity has been found to reduce the stagnant layer and enhance layer like growth of InN [8]. Smaller reactant gas flow spacing between the substrate and the quartz chamber ceiling was found to 
cause a decrease in the effective $\mathrm{NH}_{3}$ /Trimethylindium (TMIn) ratio in the growth atmosphere and enhance 2D growth [7].

In this paper we present to our knowledge the first reported data on MOVPE growth of InN by vertical closecoupled showerhead (CCS) reactor. We show that in contrast to previous reports $\mathrm{InN}$ growth is limited by the TMIn supply. In the presence of sufficient nitrogen content the growth temperature governs the island size, optical quality and electrical properties of the films.

\section{Experimental procedure}

Epitaxial films of InN were grown on c-plane sapphire substrates by a MOVPE system with a vertical CCS reactor. In the CCS design, group III and V precursors are injected into the reactor chamber through separate orifices in a showerhead injector. This should create a uniform distribution of reagent gases and prevent harmful prereactions of the precursors. TMIn and $\mathrm{NH}_{3}$ were used as indium and nitrogen precursors, respectively. To remove any impurities from the surface, the substrates were annealed at $1050{ }^{\circ} \mathrm{C}$ for $10 \mathrm{~min}$ under hydrogen flow. The substrates were then nitridated in flowing $\mathrm{NH}_{3}$ at $1050{ }^{\circ} \mathrm{C}$ for $30 \mathrm{~min}$. During the nitridation, a thin layer of aluminium nitride (AlN) forms on the sample surface [9]. After nitridation, the temperature was decreased to the growth temperature between 550 and $650{ }^{\circ} \mathrm{C}$ and the carrier gas was switched from $\mathrm{H}_{2}$ to $\mathrm{N}_{2}$ for the growth of InN. TMIn flow rate was varied between 9.1 and $36.4 \mu \mathrm{mol} / \mathrm{min}$, while ammonia flow was held constant at six SLM. This corresponds to V/III ratios between 7370 and 29500 . The thickness of InN layers varied between 400 and $1000 \mathrm{~nm}$. The reactor pressure was kept at 700 Torr during the growth.

The growth rate and layer thickness were determined insitu using an interferometric reflectance monitor system. In this technique the reflected intensity of a pulsed laser beam $(\lambda=637.8 \mathrm{~nm})$ is measured. The crystal quality of the grown InN films was analysed by high resolution $(\lambda=0.154 \mathrm{~nm})$ X-ray diffraction (XRD) measurements. The surface morphology of the films was studied with contact-mode atomic force microscopy (AFM). Photoluminescence (PL) spectra of the $\mathrm{InN}$ films were measured at $9 \mathrm{~K}$ by using argon ion laser $(\lambda=488 \mathrm{~nm})$ as the excitation source and liquid nitrogen-cooled Ge-diode as a detector. Hall measurements were used to characterize the electrical properties of the films. Non-alloyed ohmic contacts were made by conventional lift-off technique. The metals $(50 \mathrm{~nm}$ $\mathrm{Ti} / 75 \mathrm{~nm} \mathrm{Pt} / 300 \mathrm{~nm} \mathrm{Au}$ ) were deposited by electron beam deposition, as proposed by Ren et al. [10].

\section{Results and discussion}

Fig. 1 shows the growth rate of $\mathrm{InN}$ as a function of TMIn supply at various temperatures. The TMIn supply was varied from 9.1 to $36.4 \mu \mathrm{mol} / \mathrm{min}$, while the $\mathrm{NH}_{3}$ flow

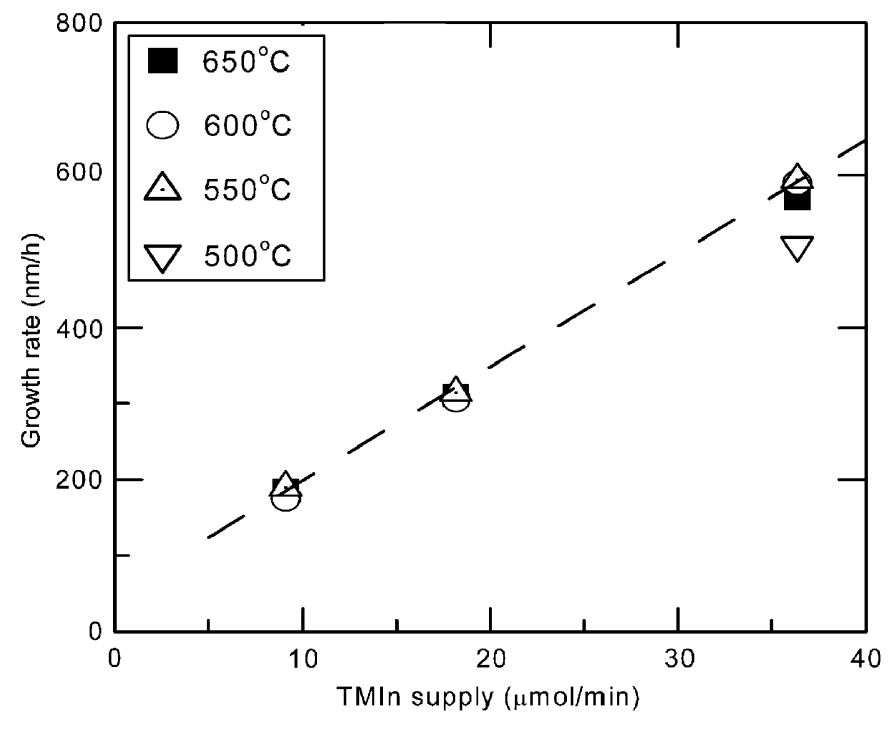

Fig. 1. Growth rate of $\mathrm{InN}$ as a function of TMIn supply at different growth temperatures.

rate was held constant. The growth rate shows a linear dependence on the TMIn flow in the studied temperature range. This indicates that the growth rate is limited by the amount of reactive indium, and not by the $\mathrm{NH}_{3}$ decomposition rate as has previously been reported [7]. These observations suggest that the cracking of $\mathrm{NH}_{3}$ at temperatures around $600{ }^{\circ} \mathrm{C}$ is enhanced in the $\mathrm{CCS}$ reactor compared with horizontal reactors. At $500{ }^{\circ} \mathrm{C}$, the growth rate decreased compared with other samples grown with the same V/III ratio. This indicates the lack of reactive nitrogen, which is caused by the sharp drop of the reaction efficiency of ammonia between 600 and $500{ }^{\circ} \mathrm{C}$ [4]. TMIn is decomposed completely at temperatures above $400^{\circ} \mathrm{C}$ [11].

Fig. 2 shows AFM scans of the InN film surface grown on nitridated sapphire substrate at 550,600 , and $650^{\circ} \mathrm{C}$ with a V/III ratio of 29500 . One can see that the film surface consists of $3 \mathrm{D}$ islands and that the island size increases with increasing growth temperature. When the growth takes place at $650^{\circ} \mathrm{C}$ the islands have a clear hexagonal base shape, but with growth temperatures of 600 and $550{ }^{\circ} \mathrm{C}$ the shape is less clear. The root mean square (RMS) surface roughness drops from 64 to $13 \mathrm{~nm}$ as the temperature is lowered from 650 to $550{ }^{\circ} \mathrm{C}$. In the literature on heteroepitaxial growth, it has been found that higher deposition rates favour layer-by-layer growth [12]. This is not observed here, the growth rate had no effect on the surface morphology in the studied range.

Fig. 3 shows a diffraction map measured from a sample grown at $600^{\circ} \mathrm{C}$ with a V/III ratio of 29500 . Diffraction peak of epitaxial InN (l 002$)$ plane is found at $2 \theta=31.4^{\circ}$, and reflection of (101) plane of metallic indium is found at $2 \theta=32.9^{\circ}$. Metallic indium crystallizes in tetragonal lattice with lattice parameters $a=b=0.325 \mathrm{~nm}$, and $c=0.495 \mathrm{~nm}$. Its $\left(\begin{array}{lll}1 & 0 & 1\end{array}\right)$ crystal plane has a nearly hexagonal atomic arrangement with in-plane atomic 

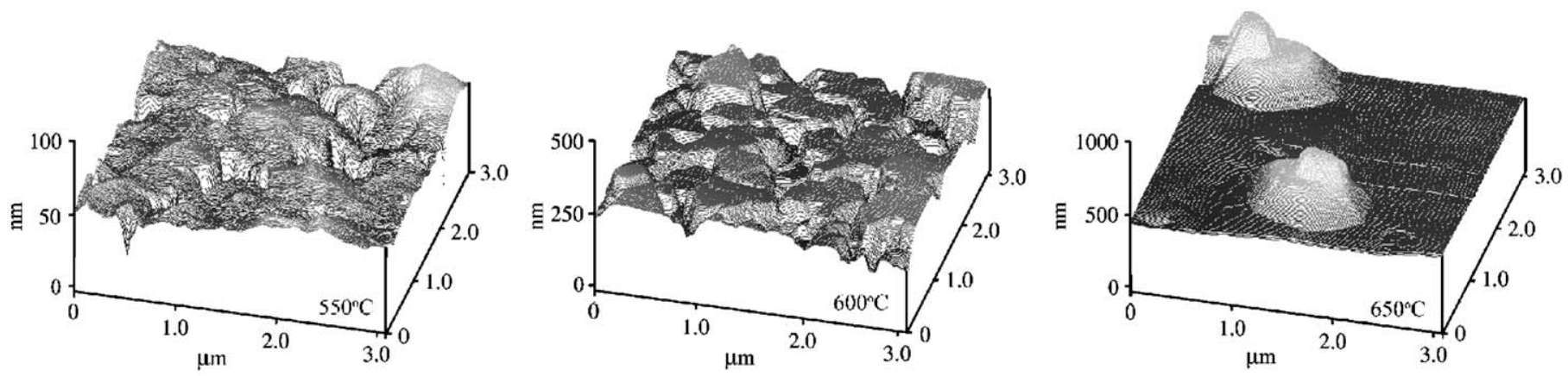

Fig. 2. AFM scans of InN film surface grown at 550,600 and $650^{\circ} \mathrm{C}$. Note the different vertical scales.

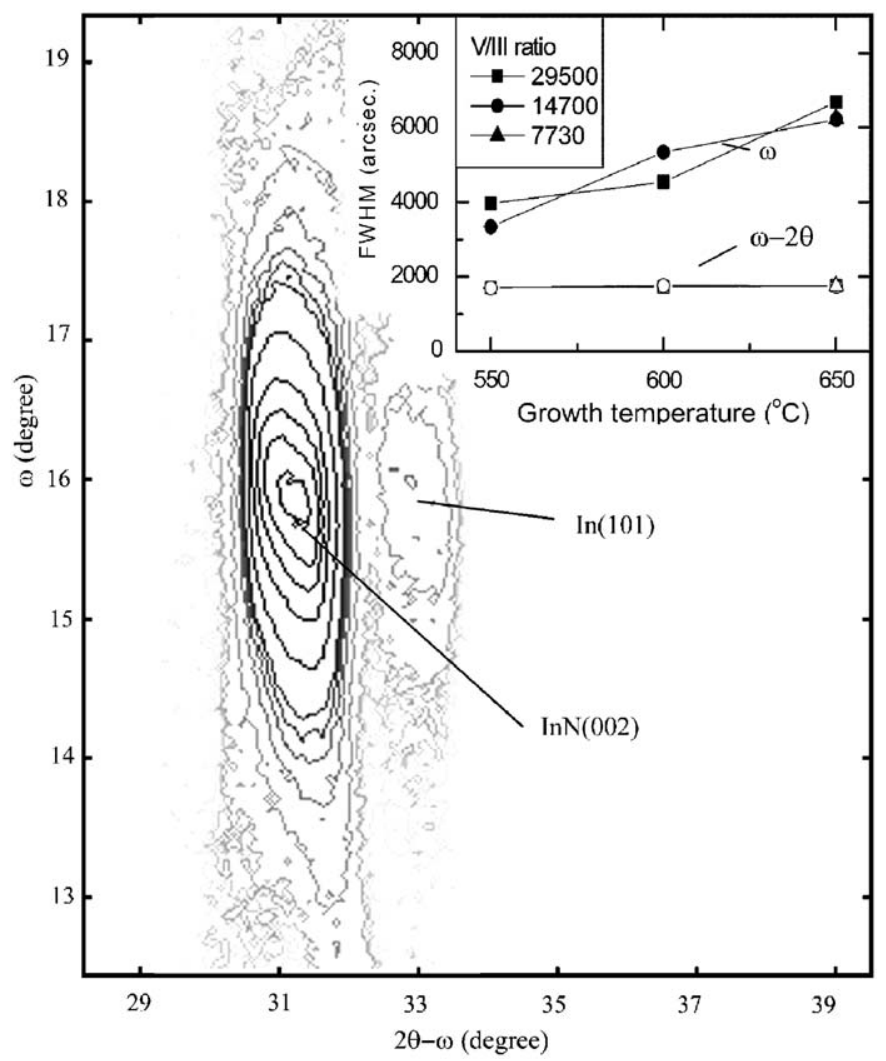

Fig. 3. X-ray diffraction map showing InN ( $\left.\begin{array}{lll}0 & 0 & 2\end{array}\right)$ and In ( $\left.\begin{array}{llll}1 & 0 & 1\end{array}\right)$ reflections. The inset shows FWHM values of $\mathrm{InN}\left(\begin{array}{ll}0 & 02\end{array}\right)$ reflection as a function of growth temperature with different V/III ratios. Open symbols denote $\omega-2 \theta$ and closed $\omega$ FWHM values.

distances that match well the lattice parameters of $\mathrm{InN}$ (0 02 2) plane [13]. The amount of metallic indium was found to increase with increasing temperature and to be independent of the V/III ratio. Threshold temperature for the formation of metallic In was between 550 and $600{ }^{\circ} \mathrm{C}$. These results indicate that the formation of metallic In is caused by the desorption of nitrogen at high temperatures, rather than by the shortage of reactive nitrogen during growth.

To evaluate the crystalline quality, full width at half maximum (FWHM) values of the XRD peaks were calculated from the $\omega-2 \theta$ and $\omega$ scans of the $\operatorname{InN}\left(\begin{array}{lll}0 & 0 & 2\end{array}\right)$ reflection. FWHM as a function of growth temperature with different V/III ratios is shown in the inset of Fig. 3. Growth temperature, growth rate or V/III ratio did not affect the $\omega-2 \theta$ FWHM values, as a FWHM of 1700 arcsec was measured from all the samples. The $\omega$ FWHM increased from 4000 to $6700 \mathrm{arcsec}$ as the temperature was increased from 550 to $650^{\circ} \mathrm{C}$. Change in the growth rate or V/III ratio did not affect the $\omega$ FWHM. In XRD measurements of symmetrical reflections, the $\omega-2 \theta$ and $\omega$ scans represent the degree of distribution of the lattice constant perpendicular to the surface and the tilt of crystalline planes, respectively [14]. These observations suggest that the films consist of domains with hexagonal single-phase crystal structure that are tilted in respect to each other. These domains cause the formation of hexagonal islands on the sample surface. Stress caused by the large lattice mismatch between the substrate and film is relaxed by dislocations formed between the islands. Increasing growth temperature results in larger domains that are more tilted in respect with each other. It is highly unlikely that the $\omega$ broadening is caused by stress, because due to the large lattice mismatch, the thick InN layer is most likely relaxed.

Fig. 4 shows InN carrier concentration and electron mobility as a function of growth temperature with various $\mathrm{V} / \mathrm{III}$ ratios. As temperature is increased from 550 to $650{ }^{\circ} \mathrm{C}$ Hall mobility increases from $100 \mathrm{~cm}^{2} /$ Vs to nearly $200 \mathrm{~cm}^{2} /$ Vs and carrier concentration drops from $6 \times 10^{20}$ to $1 \times 10^{20} \mathrm{~cm}^{-3}$. V/III ratio does not have a clear effect on either mobility or carrier concentration, so the temperature dependence does not result from more efficient cracking of $\mathrm{NH}_{3}$ but rather from better material quality resulting from the higher growth temperature. Previously enhanced decomposition of $\mathrm{NH}_{3}$ has been found to improve Hall mobility and decrease carrier concentration [15].

Fig. 5 shows PL spectra measured from samples grown at 550,600 and $650^{\circ} \mathrm{C}$. Luminescence in the range of 0.78 to $0.85 \mathrm{eV}$ was observed from all the samples. No emission was observed near the previously quoted band gap energy of $1.9 \mathrm{eV}$. This evidence of the smaller band gap energy is congruent with previous results obtained at room temperature [1]. The peak intensity showed more than tenfold increase and FWHM dropped from 133 to $37 \mathrm{meV}$ as the 


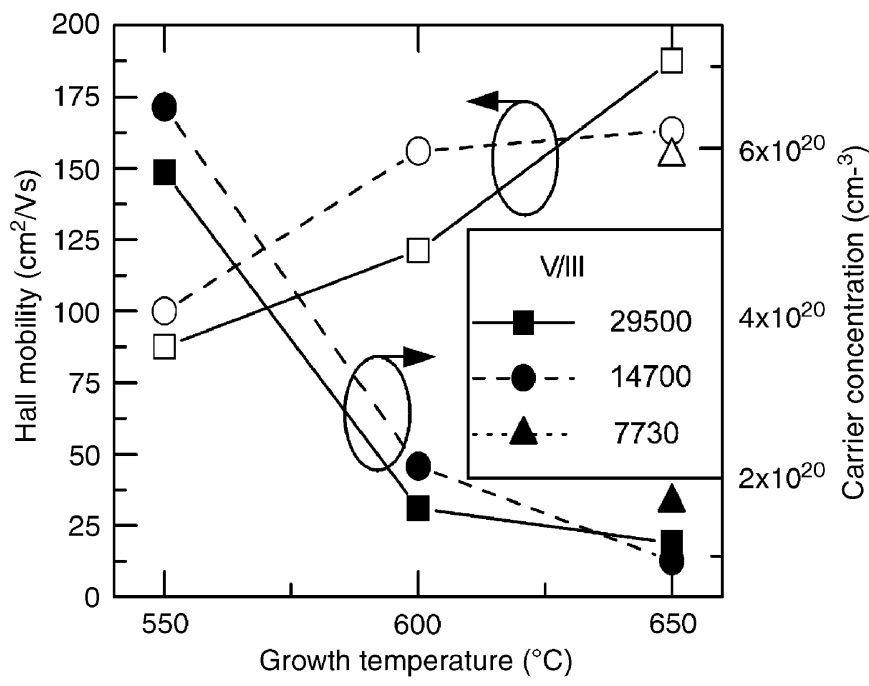

Fig. 4. Hall mobility and carrier concentration of the InN samples as a function of growth temperature with various $\mathrm{V} / \mathrm{III}$ ratios.

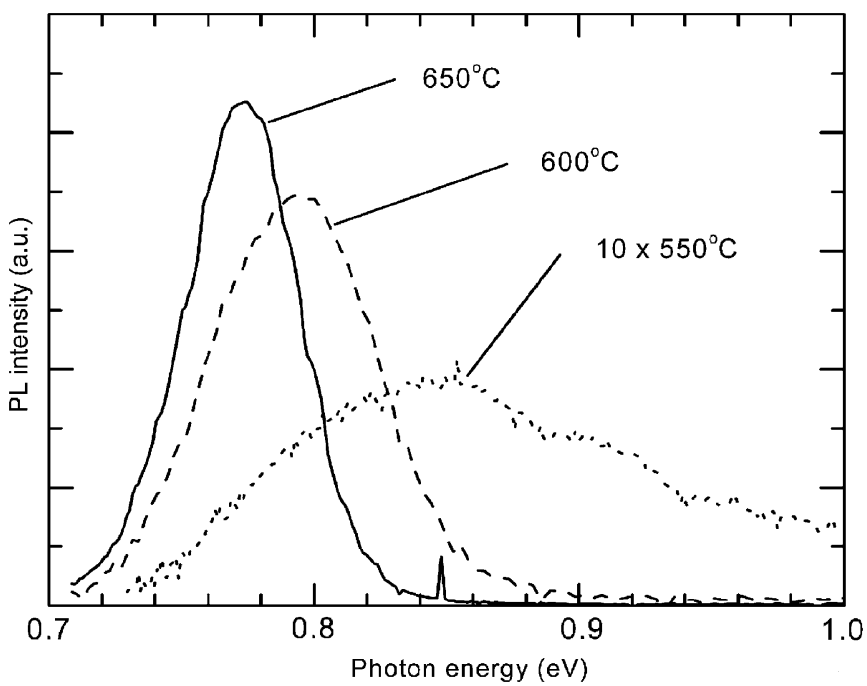

Fig. 5. PL spectra of InN samples grown at different temperatures.

growth temperature was increased from 550 to $650^{\circ} \mathrm{C}$. No change in the PL spectra was observed with different V/III ratios (spectra not shown here). The blue-shift of the PL peak energy with decreasing growth temperature is thought to result from the increase of carrier concentration in material grown at low temperature [16].

\section{Conclusions}

We have reported growth of InN on nitridated sapphire substrates by a vertical CCS reactor MOVPE. InN was found to form epitaxial layers with surface covered by islands with hexagonal base shape. Growth rate showed a linear dependence on the indium supply in the temperature range of $550-650^{\circ} \mathrm{C}$. This is clear indication of sufficient reactive nitrogen content in the reactor, and in contradiction to reports of $\mathrm{InN}$ grown by horizontal reactor MOVPE. These observations suggest that the cracking of $\mathrm{NH}_{3}$ at temperatures around $600^{\circ} \mathrm{C}$ is enhanced in the vertical CCS reactor compared to horizontal reactors.

No change in material quality was observed with different V/III ratios. In the presence of sufficient reactive nitrogen content in the reactor the growth temperature governs the electrical, optical and crystalline quality of the films. Increasing growth temperature was found to improve electrical and optical quality of the films. The formation of metallic indium above $550^{\circ} \mathrm{C}$ was found to be independent of the $\mathrm{V} / \mathrm{III}$ ratio, and In content increased with increasing growth temperature. It can be concluded that metallic indium is formed by desorption of nitrogen during growth. Photoluminescence was observed in the range of 0.78 to $0.85 \mathrm{eV}$, which supports the evidence for the band gap energy of $\mathrm{InN}$ of about $0.8 \mathrm{eV}$.

\section{References}

[1] T. Matsuoka, H. Okamoto, M. Nakao, H. Harima, E. Kurimoto, Appl. Phys. Lett. 81 (2) (2002) 1246.

[2] V.W.L. Chin, T.L. Tansley, T. Osotchan, J. Appl. Phys. 75 (1994) 7365.

[3] Q. Guo, O. Kato, A. Yoshida, J. Appl. Phys. 73 (1993) 7969.

[4] M. Mesrine, N. Grandjean, J. Massies, Appl. Phys. Lett. 72 (3) (1998) 350 .

[5] A.G. Bhuiyan, A. Yamamoto, A. Hashimoto, Y. Ito, J. Crystal Growth 236 (2002) 59

[6] S. Nakamura, G. Fasol, The Blue Laser diode, Springer, Berlin, 1997.

[7] A. Yamamoto, M. Adachi, A. Hashimoto, J. Crystal Growth 230 (2001) 351.

[8] F.-H. Yang, J.-S. Hwang, Y.-J. Yang, K.-H. Chen, J.-H. Wang, Jpn J. Appl. Phys. Part 2 (41) (2002) L1321.

[9] K. Uchida, A. Watanabe, F. Yano, M. Kouguchi, T. Tanka, S. Hinagawa, J. Appl. Phys. 79 (1996) 3487.

[10] F. Ren, C.R. Abernathy, S.J. Pearton, P.W. Wisk, Appl. Phys. Lett. 64 (1994) 1508.

[11] N.I. Buchan, C.A. Larsen, G.B. Stringfellow, J. Crystal Growth 92 (1988) 591.

[12] I. Markov, S. Stoyanov, Contemp. Phys. 28 (1987) 257.

[13] A. Krost, J. Bläsing, H. Protzmann, M. Lünenbürger, M. Heuken, Appl. Phys. Lett. 76 (11) (2000) 1395.

[14] R. Chierchia, T. Böttcher, H. Heinke, S. Einfeldt, S. Frigge, D. Hommel, J. Appl. Phys. 93 (2003) 8918.

[15] A. Yamamoto, T. Tanaka, K. Koide, A. Hashimoto, Phys. Stat. Sol. B 194 (2) (2002) 510.

[16] K. Sugita, H. Takatsuka, A. Hashimoto, A. Yamamoto, Phys. Stat. Sol. B 240 (2) (2003) 421. 